\title{
HYPOXIC INFLAMMATION, A DEADLY BRIDGE TO MALIGNANT TRANSFORMATION AND A FERTILE SOIL FOR CANCER PREVENTION
}

\author{
${ }^{1}$ Kambiz Afrasiabi, ${ }^{2}$ Robert Edwards, \\ ${ }^{3}$ Jonathan Melekh-Shalom and ${ }^{2}$ Kehui Wang \\ ${ }^{1}$ Department of Medicine, \\ ${ }^{2}$ Depatment of Pathology, \\ University of California, Irvine, Irvine, USA \\ ${ }^{3}$ American University of the Caribbean School of Medicine, Cupecoy, St. Maarten, Florida
}

Received 2013-06-12, Revised 2013-07-08; Accepted 2013-07-31

\begin{abstract}
The interplay of the inflammatory microenvironment with its hypoxic niche and the potential mechanism by which they lead to malignant transformation has long been the subject of great controversy and continues to be an area of great interest today. In our previous studies we have examined this subject by using Gia2 knock out mice as the focus of our research. These mice are well known for their tendency to develop chronic inflammation in the sub mucosa of their gut, with gradual worsening and development of colon adenocarcinoma in most as they age. It has also attracted our attention that they develop a significant increase in the number of hypoxic niches in their sub mucosa, proven by EF5 staining.In contradistinction to MSI-high colon adenocarcinomas, we have also shown that histone deacetylation rather than MLH1 promoter methylation is the main mechanism of MLH1 and MSH2 deactivation in these mice. Here we show that hypoxic niches evolve under massive selective pressure of the inflammatory microenvironment as a protective shield offering survival advantage by the up regulation of NFKb and its downstream pathways, which indeed independent of true hypoxia leads to stabilization of HIF as well, securing a dual mechanism for perpetuation and expansion of hypoxic niches. We incorporated western blot and Luciferase assay of cells exposed to hypoxia+/-inflammatory cytokines to acquire data.
\end{abstract}

Keywords: Hypoxia, Inflammation, Cancer Prevention

\section{INTRODUCTION}

Despite the fact that it has been known for a long time that chronic inflammation in the mucosa and sub mucosa of patients with inflammatory bowel disease, as well as mouse models such as Gia2 KO mouse act as harbingers for malignant transformation, until this writing, no one has been able to connect the pieces together and describe the mechanism by which malignancy arises in real life IBD patients and Gia2 KO mouse (Triantafillidis, 2009; O'Connor et al., 2010; Westbrook et al., 2010).
In patients who have been diagnosed with ulcerative colitis, prophylactic total colectomy, which is associated with significant morbidity and close surveillance is currently the mainstay of approach (Ma et al., 2012; Sandborn et al., 2009; Samuel et al., 2013). Deep understanding of the mechanism of malignant transformation might enable us to develop a much better prophylactic therapy for such patients either by reversing chronic inflammation or by breaking the bridge between chronic inflammation and malignant phenotype. Furthermore, patients that have been diagnosed with Crohn's disease undergo remitting,

Corresponding Author: Kambiz Afrasiabi, Department of Medicine, University of California, Irvine, Irvine, USA 
relapsing cycles of inflammation of different severity and duration as well and usually run a devastating natural history with variable responses to antiinflammatory and immunosuppressive agents (Colombel et al., 2010; Smith et al., 2009; Strober et al., 2010). Despite the fact that there are many different theories, ranging from genetic to environmental factors, these diseases have remained incurable thus far (Benchimol et al., 2011; Gleeson et al., 2011).

\section{MATERIALS AND METHODS}

\subsection{Luciferase Assay}

T293 cells were transfected with a CMV vector Ranilla, expressing NFKb by using conventional transfection methodology. The cells were then grown in a $10 \mathrm{~cm}$ culture dish and placed in a hypoxic chamber as well as conventional incubators with and without TNF and LPS at different concentrations and for different periods of time of up to $48 \mathrm{~h}$. NFKb expression was also measured using Luciferase assay.

\subsection{Protein Expression}

Cells were lysed according to standard protocols using protein lysis buffer to obtain protein and western blot was conducted using a SDS-page gel electrophoresis system.

\subsection{Mrna Expression}

Total RNA was extracted according to standard protocols and reverse transcribed to DNA from HT29 and CCIC spheroids which have been exposed to normoxic and $1 \%$ hypoxic conditions.

\subsection{Spheroid Formation}

By using standard stem cell medium containing EGF, b-FGF, N2 and B27, in ultra low attachment flasks, spheroids were generated out of CCIC and HT29 cells and their stemness verified using stem cell markers.

\section{RESULTS}

Chronic inflammation in the sub mucosa of our Gia2 KO mouse model leads to a significant increase in hypoxic niches, shown very clearly by EF5 immuno fluorescent staining (Marotta et al., 2011).

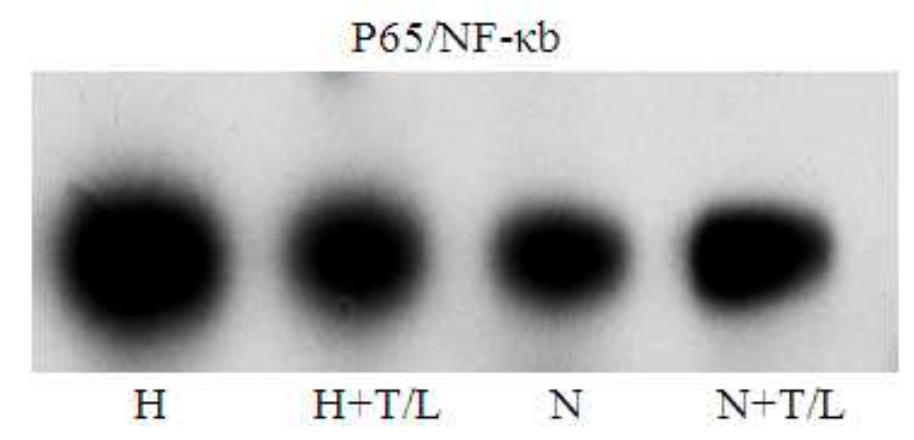

B-actin

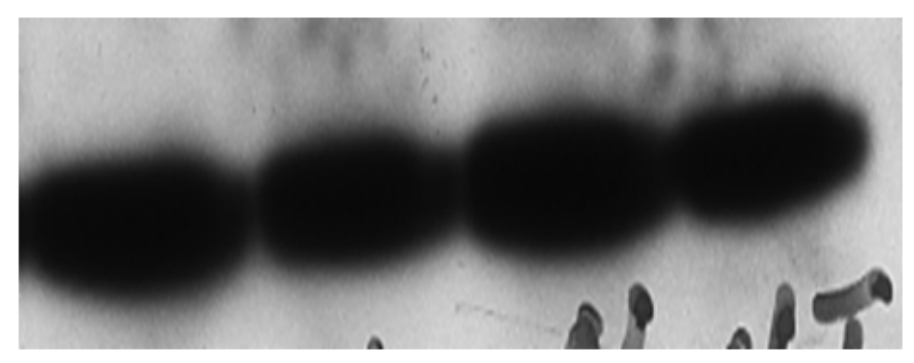

Fig. 1. p65 expression in Colon Cancer Initiating (CCIC) cells show a greater density under Hypoxic (H) conditions with TNFa and LPS (T/L) with a lower density under Normoxic (N) conditions with the same variables. B-actin was used as a loading control and confirms the integrity of protein loading 


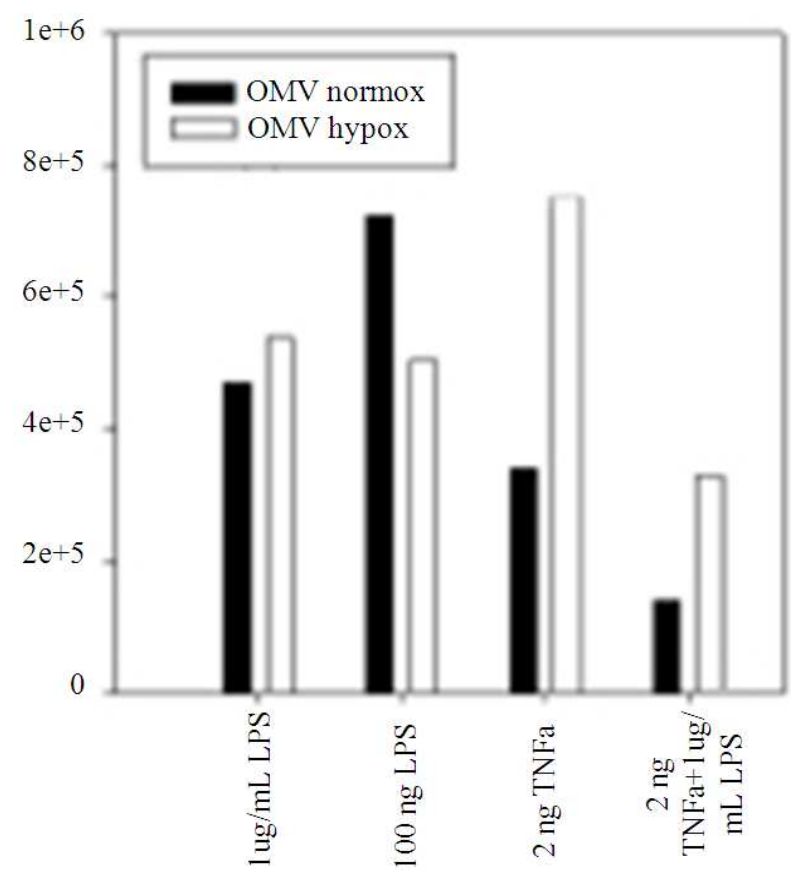

(a)

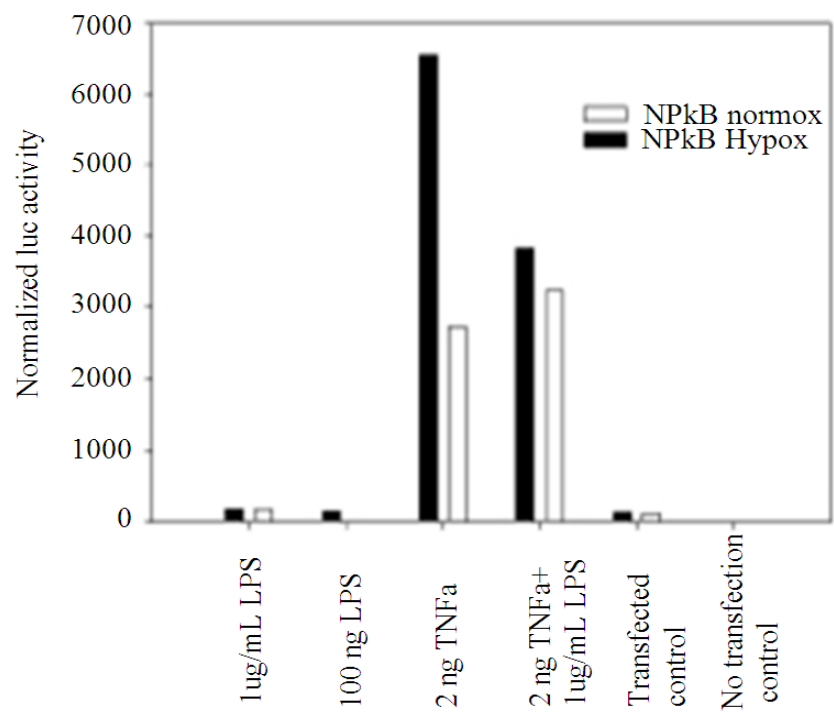

(b)

Fig. 2. The luciferase assay using the T293 cell line for transfection helps confirm our findings, demonstrating a significant upregulation of NFkb under hypoxic conditions relative to normal

In our in vitro experiments, exposure of different cell lines (T293, HT29, CCIC), to inflammatory cytokines such as TNF and LPS, with and without hypoxia led to up regulation of HIF1a (Fig. 3) and its stabilization by over expression of NFKb, as shown by western blot (Fig. 1). Hypoxia alone acted as an independent and stabilizing factor as well, which is also shown in our raw data (Table 1) and further supported by our luciferase assay (Fig. 2). To crossexamine our data, we used the HT29 cell line under similar conditions and we obtained similar results (Fig. 4). 


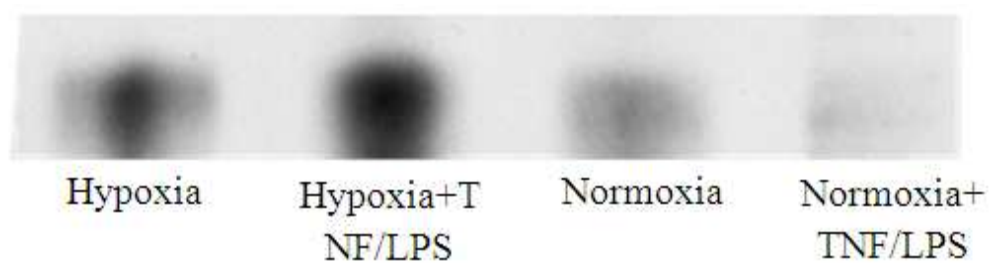

Fig. 3. CCIC exposed to hypoxiat/-THF and LPS Vs their Normoxic counterpart clearly shows upregulation of under hypoxic conditions, which increases following treatment With TNE and LPS

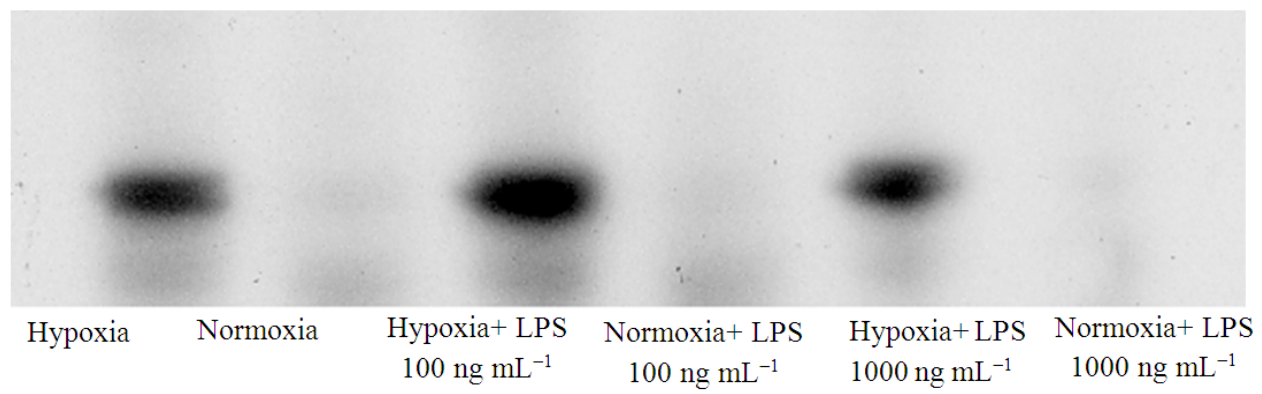

Fig. 4. p65 expression in HT29 cell line demonstrating significant overexpression in hypoxia with LPS at 100/1000 ng/ML of medium compared to normoxia under similar conditions

Table 1. Based on the raw data presented using T293 cell line, a significant change is clearly evident with TNFa under hypoxic conditions with no significant difference with LPS. NFKB expression correlates more closely with TNFa and incrementally so with increased concentration

\begin{tabular}{|c|c|c|c|}
\hline & LARII & $\mathrm{S}$ and $\mathrm{G}$ & Ratio \\
\hline Hypox 48 hrs alone & 321.600 & 2.526 & 127.32 \\
\hline Then hypox $48+10 \mathrm{ng} \mathrm{mL}^{-1}$ LPS & 1430.000 & 4.644 & 307.92 \\
\hline Then hypox $48+100 \mathrm{ng} \mathrm{mL}^{-1} \mathrm{LPS}$ & 1080.000 & 4.033 & 267.79 \\
\hline Then hypox $48+2 \mathrm{ng} \mathrm{mL}^{-1} \mathrm{TNFa}$ & 3009.000 & 4.988 & 603.25 \\
\hline Then hypox $48+10 \mathrm{ng} \mathrm{mL}^{-1} \mathrm{TNFa}$ & 1751.000 & 0.992 & 1765.12 \\
\hline Hypoxia 48 hrs no transfection control & 0.058 & 0.250 & 0.23 \\
\hline Normoxia 48 hrs alone & 1737.000 & 12.830 & 135.39 \\
\hline Then normoxia $48+10 \mathrm{ng} \mathrm{mL}^{-1}$ LPS & 448.000 & 1.418 & 315.94 \\
\hline Then normoxia $48+100 \mathrm{ng} \mathrm{mL}^{-1} \mathrm{LPS}$ & 2120.000 & 9.705 & 218.44 \\
\hline Then normoxia $48+2 \mathrm{ng} \mathrm{mL}^{-1} \mathrm{TNFa}$ & 1599.000 & 4.399 & 363.49 \\
\hline Then normoxia $48+10 \mathrm{ng} \mathrm{mL}^{-1} \mathrm{TNFa}$ & 1206.000 & 1.573 & 766.69 \\
\hline Normoxiaia $48 \mathrm{hrs}$ no transfection control & 0.020 & 0.270 & 0.07 \\
\hline
\end{tabular}

Hypoxia acted independently and synergistically with TNF and/or LPS, as far as over expression of NFKb is concerned, thus securing a dual mechanism of survival under harsh inflammatory micro environmental stimuli.

\section{DISCUSSION}

Despite the fact that chronic inflammation in different organs has been found to be associated with malignant transformation, ranging from adnexal/lacrimal gland maltomas in response to
Chlamydia Trachomati (Kram et al., 2010) to thyroid lymphoma of Hashimoto's thyroiditis (Rapoport and McLachlan, 2012) gastric maltoma secondary to Helicobacter Pylori (Pervez et al., 2011), to hepatocellular carcinoma secondary to chronic hepatitis B and C (El-Serag, 2012; Lok et al., 2009), colon adenocarcinoma due to ulcerative colitis (Jess et al., 2012), EBV and Papilloma virus related Burkitt's lymphoma and squamous cell carcinoma of head and neck as well as cervix (Lajer et al., 2012; Guan et al., 2012) and most recently implications of chronic 
inflammation in some leukemias (Chen et al., 2010), until this writing no one has shown the detailed dynamics and interplay of chronic inflammation with malignant transformation. Here we clearly show that chronic inflammation triggers the formation and expansion of hypoxic niches, which come into existence as a protective shield in response to the high selective pressure generated by harsh inflammatory microenvironment. We further define that convergence of hypoxia and chronic inflammation would offer the dual survival advantage to these cells by upregulation of NFKb and stabilization of hypoxic niches through up regulation of HIF in a synergistic fashion. It also been widely demonstrated that the activation of pathways such as FOXO3a would add further to the preservation of the hypoxic niche, which would become the nest for nurturing cells with stemness capabilities, supported by spheroid formation and stem cell marker expression (Bakker et al., 2007). Clearly the probability of malignant transformation would increase dramatically in these massively expanded hypoxic niches, through random genetic events, as well as the direct and indirect effects of exposure to environmental carcinogens.

Our model allows for early/pre-emptive intervention with either anti-inflammatory agents and perhaps by recruiting genetically engineered cells into either the inflammatory microenvironment or hypoxic niches to generate anti-inflammatory proteins or oxygen respectively.

This model also allows a new scoring system for measurement of success of our new approach to these old problems by serial biopsies and staining for either the markers of hypoxia, EF5 or inflammation by immunostaining for TNF or LPS.

As of today our biomedical society has learned that eradication of $H$. pylori could lead to reversal and prevention of gastric maltomas, eradication of hepatitis $\mathrm{B}$ and $\mathrm{C}$ would lead to prevention of hepatocellular carcinoma, vaccination against the prevalent serotypes of papilloma virus could lead to prevention of squamous cell carcinoma of cervix. In the near future we should be able to send our genetically engineered cells by using nanodelivery mechanisms to the birthplace of malignancy, namely the inflammatory mficroenvironment and hypoxic niches on a selective basis.

\section{CONCLUSION}

We have discovered that the generation and expansion of hypoxic niches in chronic inflammatory conditions is a response to the deadly inflammatory microenvironment. This "protective shield" ultimately nurtures cells with stemness capabiltiies and promotes malignant transformation. Furthermore, our findings could generate a clinical platform for a future scoring system that could be used for the assessment of novel preventive and therapeutic measures.

\section{REFERENCES}

Bakker, W.J., I.S. Harris and T.W. Mak, 2007. FOXO3a is activated in response to hypoxic stress and inhibits HIF1-induced apoptosis via regulation of CITED2. Mol. Cell, 28: 941-953. DOI: 10.1016/j.molcel.2007.10.035

Benchimol, E.I., K.J. Fortinsky, P. Gozdyra, M.V.D. Heuvel and J.V. Limbergen et al., 2011. Epidemiology of pediatric inflammatory bowel disease: A systematic review of international trends. Inflammatory Bowel Dis., 17: 423-439. PMID: 20564651

Chen, L.S., K. Balakrishnan and V. Gandhi, 2010. Inflammation and survival pathways: Chronic lymphocytic leukemia as a model system. Biochem. Pharmacol., 80: 1936-1945. DOI: 10.1016/j.bcp.2010.07.039

Colombel, J.F., W.J. Sandborn, W. Reinisch, G.J. Mantzaris and A. Kornbluth et al., 2010. Infliximab, azathioprine, or combination therapy for Crohn's disease. New Eng. J. Med., 362: 1383-1395. DOI: 10.1056/NEJMoa0904492

El-Serag, H.B., 2012. Epidemiology of viral hepatitis and hepatocellular carcinoma. Gastroenterology, 142: 1264-1273. DOI: 10.1053/j.gastro.2011.12.061

Gleeson, M., N.C. Bishop, D.J. Stensel, M.R. Lindley and S.S. Mastana et al., 2011. The antiinflammatory effects of exercise: Mechanisms and implications for the prevention and treatment of disease. Nature Rev. Immunol., 11: 607-615. DOI: 10.1038/nri3041

Guan, P., R.H. Jones, N. Li, L. Bruni, S. De Sanjose and S. Franceschi et al., 2012. Human papillomavirus types in 115,789 HPV-positive women: A metaanalysis from cervical infection to cancer. Int. J. Cancer, 131: 2349-2359. DOI: 10.1002/ijc.27485

Jess, T., C. Rungoe and L. Peyrin-Biroulet, 2012. Risk of colorectal cancer in patients with ulcerative colitis: A meta-analysis of population-based cohort studies. Clin. Gastroenterol. Hepatol., 10: 639-645. DOI: 10.1016/j.cgh.2012.01.010 
Kram, D.E., C.D. Brathwaite and Z.A. Khatib, 2010. Bilateral conjunctival extranodal marginal zone Bcell lymphoma. Pediatric Blood Cancer, 55: 14141416. DOI: $10.1002 /$ pbc. 22694

Lajer, C.B., E. Garnaes, L. Friis-Hansen, B. Norrild and M.H. Therkildsen et al., 2012. The role of miRNAs in Human Papilloma Virus (HPV)-associated cancers: Bridging between HPV-related head and neck cancer and cervical cancer. Briti. J. Cancer, 106: 1526-1534. DOI: 10.1038/bjc.2012.109

Lok, A.S., L.B. Seeff, T.R. Morgan, A.M. Di Bisceglie and R.K. Sterling et al., 2009. Incidence of hepatocellular carcinoma and associated risk factors in hepatitis C-related advanced liver disease. Gastroenterology, 136: 138-148. DOI: 10.1053/j.gastro.2008.09.014

Ma, C., M. Crespin, M.C. Proulx, S. DeSilva and J. Hubbard et al., 2012. Postoperative complications following colectomy for ulcerative colitis: A validation study. BMC Gastroenterol., 12: 39-39. DOI: 10.1186/1471-230X-12-39

Marotta, D., J. Karar, W.T. Jenkins, M. Kumanova and K.W. Jenkins et al., 2011. In vivo profiling of hypoxic gene expression in gliomas using the hypoxia marker EF5 and laser-capture microdissection. Cancer Res., 71: 779-789. DOI: 10.1158/0008-5472.CAN-10-3061

O'Connor, P.M., T.K. Lapointe, P.L. Beck and A.G. Buret, 2010. Mechanisms by which inflammation may increase intestinal cancer risk in inflammatory bowel disease. Inflamm. Bowel Dis., 16: 1411-1420. PMID: 20155848

Pervez, S., N. Ali, H. Aaqil, K. Mumtaz and S.S. Ullah et al., 2011. Gastric MALT lymphoma: A rarity. J. College Phys. Surgeons Pak., 21: 171-172. PMID: 21419026
Rapoport, B. and S.M. McLachlan, 2012. Thyroid Lymphoma. Thyroid Cancer, 30: 201-205. DOI: 10.1007/978-1-4614-0875-8_11

Samuel, S., S.B. Ingle, S. Dhillon, S. Yadav and W.S. Harmsen et al., 2013. Cumulative Incidence and Risk Factors for Hospitalization and Surgery in a Population-based Cohort of Ulcerative Colitis. Inflamm. Bowel Dis. 9: 1858-1866. DOI: 10.1097/MIB.0b013e31828c84c5

Sandborn, W.J., P. Rutgeerts, B.G. Feagan, W. Reinisch and A. Olson et al., 2009. Colectomy rate comparison after treatment of ulcerative colitis with placebo or infliximab. Gastroenterology, 137: 12501260. DOI: 10.1053/j.gastro.2009.06.061

Smith, A.M., F.Z. Rahman, B.H. Hayee, S.J. Graham, D.J. Marks and A.W. Segal et al., 2009. Disordered macrophage cytokine secretion underlies impaired acute inflammation and bacterial clearance in Crohn's disease. J. Exp. Med., 206: 1883-1897. DOI: $10.1084 /$ jem.20091233

Strober, W., F. Zhang, A. Kitani, I. Fuss and S. FichtnerFeigl, 2010. Pro-Inflammatory cytokines underlying the inflammation of crohn's disease. Current Opin. Gastroenterol., 26: 310-317. DOI: 10.1097/MOG.0b013e328339d099

Triantafillidis, J.K., G. Nasioulas and P.A. Kosmidis, 2009. Colorectal cancer and inflammatory bowel disease: Epidemiology, risk factors, mechanisms of carcinogenesis and prevention strategies. Anticancer Res., 29: 2727-2737. PMID: 19596953

Westbrook, A.M., A. Szakmary and R.H. Schiestl, 2010. Mechanisms of intestinal inflammation and development of associated cancers: Lessons learned from mouse models. Mutation Res. Rev. Mutation Res., 705: 40-59. DOI: 10.1016/j.mrrev.2010.03.001 